EMSP Final Report, December 2005

\title{
Physical Characterization of Solid-Liquid Slurries at High Weight Fractions Using Optical and Ultrasonic Methods
}

\author{
This material is based upon work supported by the U S. Department of Energy \\ under Award No. DE-FG07-01ER63267 \\ Project Number: 81964
}

\author{
Lead Principal Investigators: \\ L.W. Burgess and A.M. Brodsky \\ Center for Process Analytical Chemistry \\ University of Washington \\ Seattle, Washington \\ (206) 543-0579 \\ Lloyd@cpac.washington.edu \\ Co-Investigators: \\ P.D. Panetta, B. Tucker, and K. Denslow \\ Pacific Northwest National Laboratory \\ Richland, Washington \\ (509) 372-6107 \\ Paul.Panetta@pnl.gov \\ Graduate Student: \\ S.L. Randall \\ Center for Process Analytical Chemistry \\ University of Washington \\ Seattle, Washington \\ (206) 616-1492 \\ sr33@u.washington.edu
}

\section{Executive Summary}

Remediation of highly radioactive waste is a major technical and programmatic challenge for the DOE. Rapid, on-line physical characterization of highly concentrated slurries is required for the safe and efficient remediation of 90 million gallons of high level radioactive waste (HLW), sodium bearing waste, and mixed waste. The research presented here, describes a collaborative effort between Pacific Northwest National Laboratory (PNNL) and the University of Washington (UW) to directly address the need for rapid on-line characterization of the physical properties of HLW slurries during all phases of the remediation process, from in-tank characterization of sediments to monitoring of the concentration, particle size, and degree of agglomeration and gelation of slurries during transport. Near-surface characterization of the slurry flow in the particle size range from nanometer to micrometer is examined using optical low coherence reflectometry. Volumetric characterization at depths in the slurry flow, up to several 
centimeters in the particle size range from the micrometer to millimeter, is realized by utilizing ultrasonic backscatter and diffuses fields. One of the strengths, the teaming up of significant talents in both experimental and theoretical optics and in ultrasonics, provides a synergistic approach to integrate these complimentary techniques. One of the benefits of this combined approach is the physical characterization of HLW over a concentration and particle size range that is broader than can be achieved with today's technology. This will avoid a costly increase in waste stream volume due to excess dilution, and will lessen chance of plugging pipes that could shut down expensive processing lines.

\section{Research Objectives}

The project has received continuing project support under DE-FG02-05ER63990. This report represents progress during the initial funding period and a subsequent no cost extension which aided us in bridging a gap (approximately six months) between the initial funding period and the beginning of the renewal period.

Slurry systems with mean particle sizes from $\sim 0.01$ um to 800 um have been studied at concentration ranging from very dilute ( $<1 \mathrm{wt} \%)$ to $40 \mathrm{wt} \%$. The interrelationships between the attenuation, the direct backscattered field, and the diffusely propagating field have been investigated. These fields are rich with information and unraveling their interrelationships provides the robust methodology needed to characterize these HLW slurries and enhance the clean up process at the DOE facilities.

There are three tasks: 1) develop new optical and acoustic scattering measurements to provide the fundamental science needed for successful device development and implementation, 2) develop theories that describe the interrelationship between wave propagation and the physical properties of the slurry, and 3) perform inversions of the theories and compare them with the experimental measurements to non-intrusively characterize slurries.

The integration of both optic and ultrasonic techniques has been unique and exciting because it offers a combined methodology that exploits the best features of these two powerful techniques. This combination of capabilities has allowed characterization to be performed over a broader range of particle sizes and concentrations than can be accomplished with existing techniques.

\section{Optical Measurements}

The optical measurements are based on the investigation of slurries with Optical LowCoherence Reflectometry (OLCR). OLCR is a white-light interferometric technique that incorporates a broadband light source with a classical Michelson interferometer. In previous works ${ }^{1-3}$ we have demonstrated that OLCR is an effective tool for nondestructive analysis of multi-layer and multi-scattering systems. Initial work was performed using a Hewlett-Packard OLCR instrument and evolved to use the more flexible Optiphase instrument which has undergone a great degree of development during this project period. The probe development has been a non-trivial aspect of the instrumentation. The 
instrument design requires a triggering reflection, which we have chosen to be from the fiber end face without any contact from the sample. By adding the non-contact
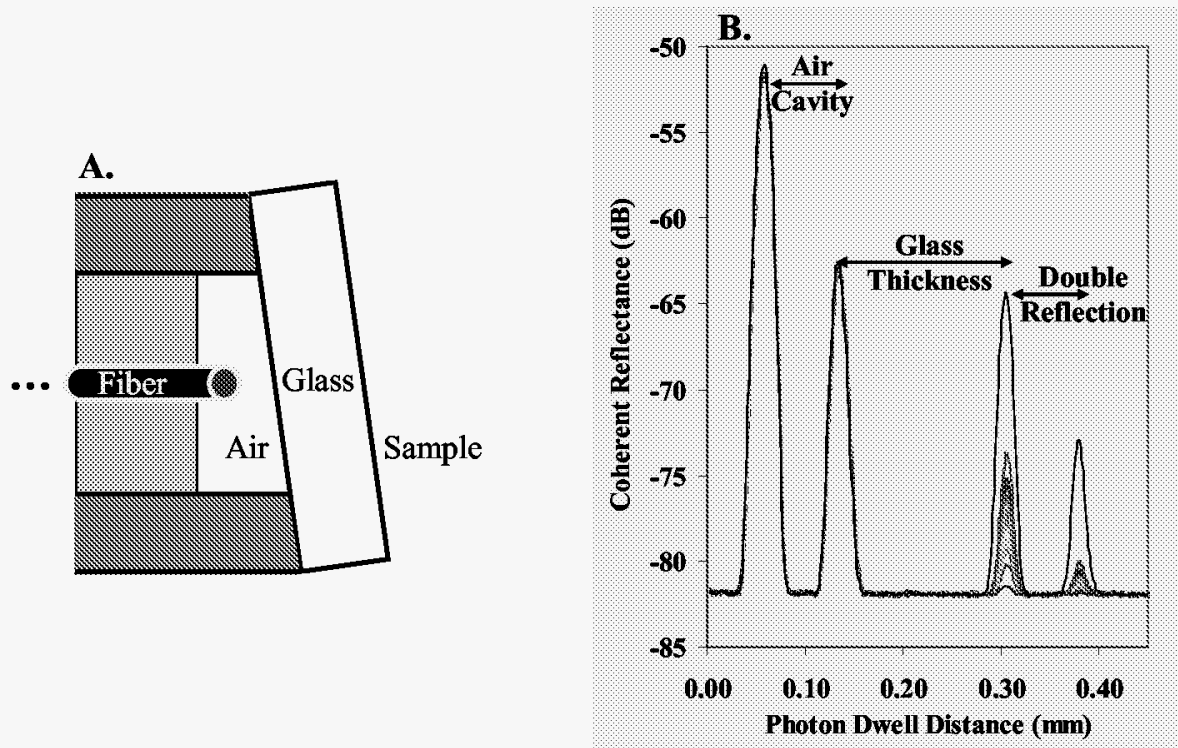

Figure 1. A) Simplified probe design consisting of bare single-mode fiber, the air cavity, and the glass window at an 8 degree angle. B) The resulting four reflections from a series of solvents with varying refractive indices.

limitation, the fiber end face reflection can be used not only to trigger the instrumentation, but also to serve as a reference to correct for source fluctuations and allows us to track the matrix refractive index. For solid-liquid slurries, the probe has been designed to consist of standard single mode optical fiber, followed by a small air gap, and then a glass window angled at $8^{\circ}$ relative to the fiber end face. This design results in triggering and reference reflections from the interface between the fiber end face and air, and the interface between air and the inside surface of the glass window. The reflection resulting from the outside surface of the glass window, which is in contact with the sample, will vary in amplitude based upon the refractive index difference between the window and the sample. The probe design is illustrated as Figure 1 and the resulting OLCR profiles from the solvent series are shown in Figure 1B. The fourth reflection is a resonance effect which occurs due to the length of the air cavity within the probe. In later iterations, the air cavity length was decreased and the resonance reflection disappeared. The amplitude ratio of the reflection from the air-inside window interface to the reflection of the outside window-sample interface can be related to the refractive index of the sample. In the Rayleigh scattering regime, the refractive index can be directly related to the concentration of dispersed material of known refractive index, and can be diagnostic during agglomeration as particles transition from one scattering regime to the other. This feature aids us in deconvoluting dynamic changes in the slurry system.

In the optical experimental work we have used standard monodispersed polystyrene nanospheres purchased from Duke Scientific Corporation to act as a model system for theory and experimental development over a large dynamic range of particle sizes. The 
shape and intensity of the OLCR profiles changes as the measured particle size transitions from the Rayleigh regime to the Fraunhofer regime, as shown in Figure 2. The spike at photon dwell distances around $3 \mathrm{~mm}$ are due to a source artifact and are not related to the sample.

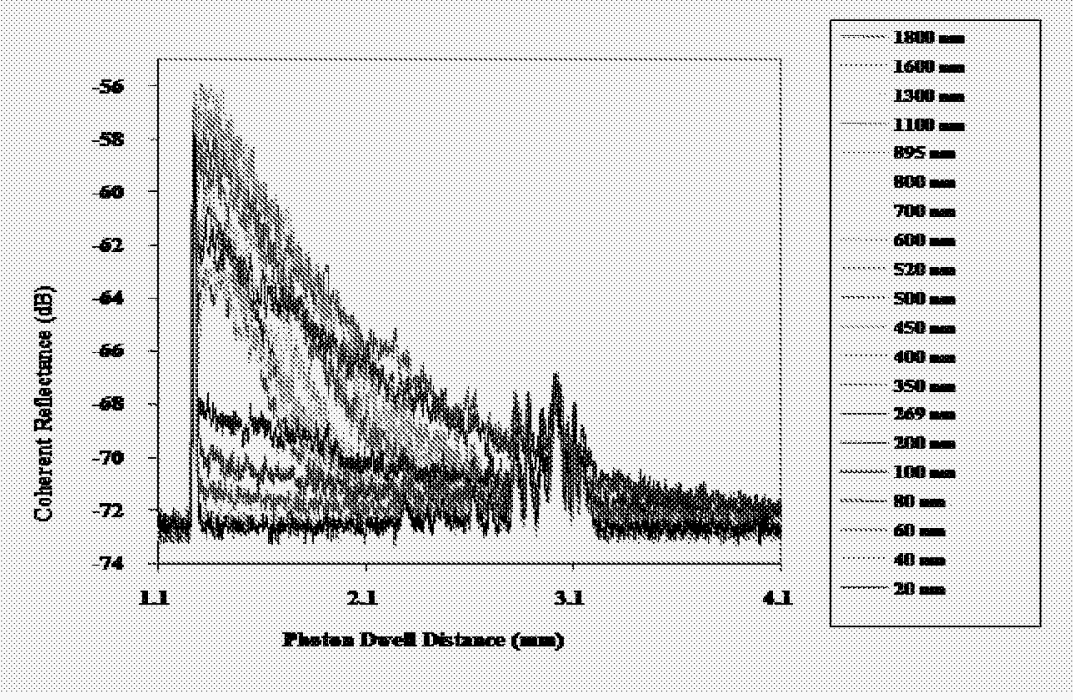

Figure 2. OLCR profiles for polystyrene sphere standards for diameters $20 \mathrm{~nm}$ to $1745 \mathrm{~nm}$.

One of the most interesting results of this work is the observation of so-called Mie resonances when the particle radii of a randomly distributed system are comparable with the wavelength of light. This non-monotonic oscillation of backscattered intensity is dependent upon optical parameters, such as dielectric contrast between the medium and the particles.

Advances in the optical theory of multiple scattering have been made, specifically to begin to extract information pertaining to system properties, such as particle size, concentration, and polydispersity, from all regions of the OLCR profile. Theoretical estimations of the experimental amplitude oscillation are described in the following equation:

$$
\begin{aligned}
\mathrm{S}-\mathrm{S} 0=\mathrm{C} \rho 0[0.25+1 / \mathrm{y} 2 & +2(1-\cos (\mathrm{y})) / \mathrm{y} 4-\sin (\mathrm{y}) / \mathrm{y}+(1-\cos (\mathrm{y})) / \mathrm{y} 2-2 \sin \mathrm{y} / \mathrm{y} 3] \\
& \rightarrow \mathrm{C} \rho 0(\mathrm{R} / \lambda) 2(4 \pi \Delta \mathrm{nM} 2 / 3) 2 \\
& \text { for } \mathrm{R} \Delta / \lambda<<1 \text { (Rayleigh Limit) }
\end{aligned}
$$

where $S$ is the total signal, $S_{0}$ is a combination of an adjustment for the baseline signal and an indication of the non-uniformity of the solution, $\mathrm{C}$ is an instrumentally-based constant, $\rho_{0}$ is the volume fraction of particles in the studied media, $y=4 \pi R \Delta \varepsilon_{\mathrm{M}} / \lambda$ with $\mathrm{R}$ as the particle radius, $\Delta$ as the optical contrast between the particles and media, $\varepsilon_{\mathrm{M}}$ is the dielectric function of the media, and $\lambda$ as the wavelength of light in a vacuum. The described results are interesting as a step toward advancement in the understanding of the 
theoretically and practically important problem of wave propagation in multiscattering random media.

Analysis of two polystyrene suspension series with the same range of particle sizes but different volume fractions led to a few interesting comparisons. The calculated effective refractive indices of both suspensions were equal, $1.3585 \mathrm{RI}$ units, based upon the calibration established by the peak ratios from the solvent series described above. The theoretical expression as described in the sections above suggested that an increase by a factor of ten in volume fraction led to an increase by a factor of approximately ten for the amplitude of Mie resonances, and this was confirmed by comparison of experimental data. The two experimental Mie resonance curves from the $1 \%(\mathrm{v} / \mathrm{v})$ and $10 \%(\mathrm{v} / \mathrm{v})$ suspension series is shown as Figure 3. A manuscript has been published to describe both the experimental and theoretical Mie resonance results ${ }^{4}$.

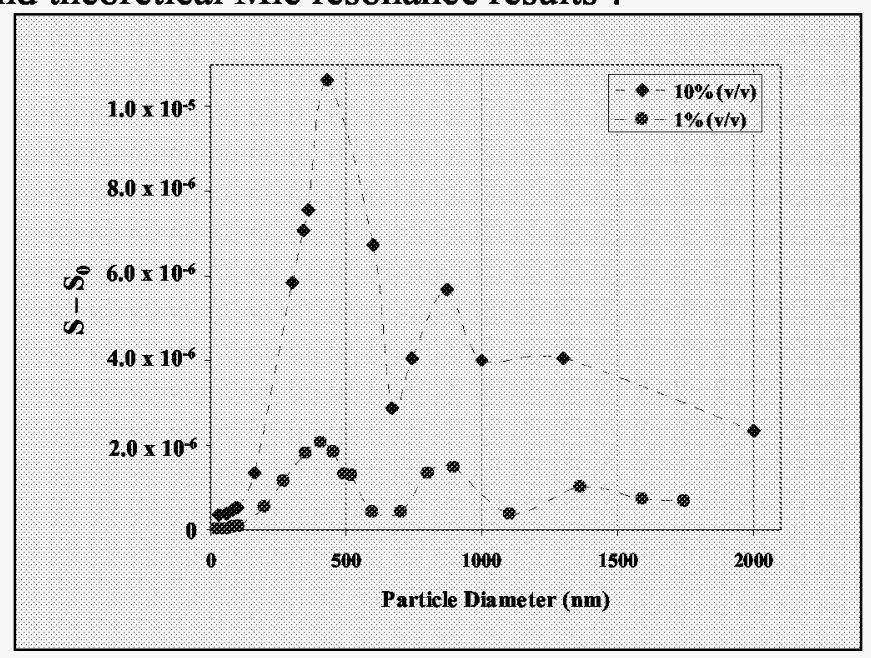

Figure 3. Comparison of experimental Mie resonance curves from a $1 \%(\mathrm{v} / \mathrm{v})$ polystyrene suspension series (circles) and a $10 \%(\mathrm{v} / \mathrm{v})$ polystyrene suspension series.

Both standard polystyrene spheres and DOE waste simulants have been used for polydispersed measurements. We have reported on OLCR measurement capabilities in a more complex and realistic matrices including bimodal mixtures of individual polydispersed alumina suspensions. These were easily distinguishable from each other when measured individually at $30 \mathrm{wt} \%$, and the trend of changing average particle size can be easily followed through the profile amplitude. This result is very exciting and extremely encouraging towards our ability to track changing dimensions in a complex, polydispersed, multi-modal surrogate slurry. The alumina component will serve as an excellent surrogate waste model for both static and dynamic studies.

We have developed a greater understanding of how the complete surrogate slurry will act through the analysis of bimodal monodispersed polystyrene mixtures. Bimodal polystyrene solutions were prepared by mixing monodispersed $1 \% \mathrm{w} / \mathrm{w}$ standards in varying amounts to form the desired average size or size ratios as shown in the table in Figure 4. The five bimodal samples were analyzed which had a mean particle size of 60 $\mathrm{nm}$, but different compositions. The experimental method used was consistent with that from the standard monodispersed systems. The decay curves of the resulting OLCR profiles are very similar in intensity and slope and clear relationships between the 
contributions from the bimodal components and the regions of the profiles cannot be easily determined.

Differences between the bimodal system composition arise however, when the fluctuations of an individual signal from the mean are analyzed throughout the entire OLCR profile. These fluctuations are not noise-based and do not average out with multiple scans in the same location of a solid sample. Rather, they are due to fixed heterogeneities within the system and contain information regarding particle clustering. The C-value for fluctuation analysis is calculated by

$$
\mathrm{C}=<\mathrm{J}(\mathrm{L}) \mathrm{J}(\mathrm{L}+\Delta \mathrm{L})>/<\mathrm{J}(\mathrm{L}+\Delta \mathrm{L})><\mathrm{J}(\mathrm{L})>-1
$$

where $\mathrm{J}(\mathrm{L})$ is the OLCR signal at a wavepacket dwell distance of $\mathrm{L}$ and $\Delta \mathrm{L}$ is indicative of the step size or correlation distance in $\mathrm{mm}$. Log $\mathrm{C}^{2}$ is plotted for each $\Delta \mathrm{L}$, or correlation distance in $\mathrm{mm}$. Figure 4 illustrates that with increased correlation distance, the distinction between the samples of the various compositions becomes more pronounced. First, variation in the signal is measured over each scan from the mean over the decay length of the profile. Then, by 'stepping through' the OLCR profiles and correlating the intensity of individual data points to their neighbors', a sense of a correlation distance, $\Delta \mathrm{L}$, is found, which is related to the composition of the matrices. Fluctuations are especially important for monitoring phase transitions, such as gelation. The OLCR profile of a liquid solution is relatively smooth, due to Brownian motion causing an averaging of the signal fluctuations. However, as a sample transitions into a more solid state, Brownian motion slows and the fluctuations in the OLCR profile increase.
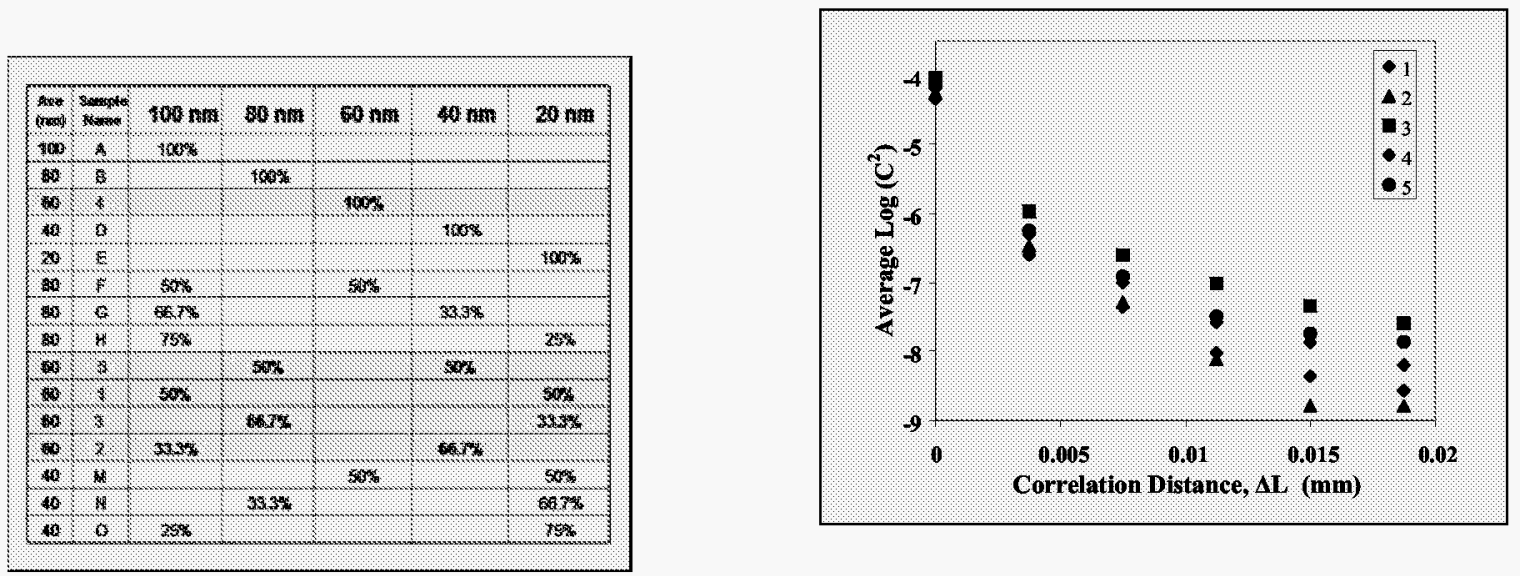

Figure 4. The experimental design and a figure illustrating how the differentiation between bimodal suspensions (indicated in red) increases with increasing correlation distance.

However, as a sample transitions into a more solid state, Brownian motion slows and the fluctuations in the OLCR profile increase. This is because only limited numbers of random particle distributions are available for analysis by the wavepacket. When the system under test becomes solid, only one distribution is available and the fluctuations 
will become very stable. Gelation, agglomeration, and sedimentation have all been shown to occur by adjusting parameters of the alumina system (i.e. $\mathrm{pH}$, flow rate, temperature) and are being systematically studied in ongoing work.

Overall, we have made significant progress in the analysis of signals obtained from multiple optical scattering events. We have observed the appearance of Mie resonances, a non-monotonic oscillation of backscattered intensity dependent on optical parameters, over the interval where radii are comparable to the wavelength. The results above clearly indicate that it is possible to extract important static and dynamic parametric information from dense scattering systems with OLCR. Between the refractive index information, fluctuation distributions, amplitude variation, and decay rate, we should be able to extract characteristic information about particle systems. If all the variables are changing simultaneous, some resolution of the parameter convolutions can be addressed by utilizing the synergy of the project and incorporating information from the ultrasound data.

These data do not yet reveal how we may be able to extract effects such as gross polydispersity, particle geometry, variable viscosity, or widely variable dielectric contrast that will be present in the slurries and colloidal systems of interest to the DOE. Our ongoing challenge will be to select well-characterized surrogate systems that will allow us to study these processes and aid in further theoretical development.

\section{Ultrasonic Measurements}

PNNL has been developing ultrasonic measurement methodologies to characterize solid liquid suspensions at high concentrations. A schematic of the measurement system is shown in Figure 5a with representative signal used for calculating the attenuation and speed of sound in Figure 5b, the backscattering in Figure 5c, and the diffuse field in Figure 5d. The measurement cell is composed of a ring with opposing transducers to send and receive the ultrasonic fields. The speed of sound is simply a measure of the transit time across the chamber and the attenuation is a measure of the amount of energy lost due to scattering and absorption as the sound traverses the chamber. The backscattering is a measure of the amount of energy that is returned to the transmitting transducer. The diffuse field develops after the ultrasonic field has undergone many scattering events with the particles in the fluid and the walls of the chamber. For the attenuation, backscattering and the diffuse field, analysis is performed to produce an ultrasonic response as a function of frequency. The effects from the measurement system and the transducer are removed from the received signal by utilizing an appropriate reference signal. Details can be found in publications that resulted from this work. 
(a)

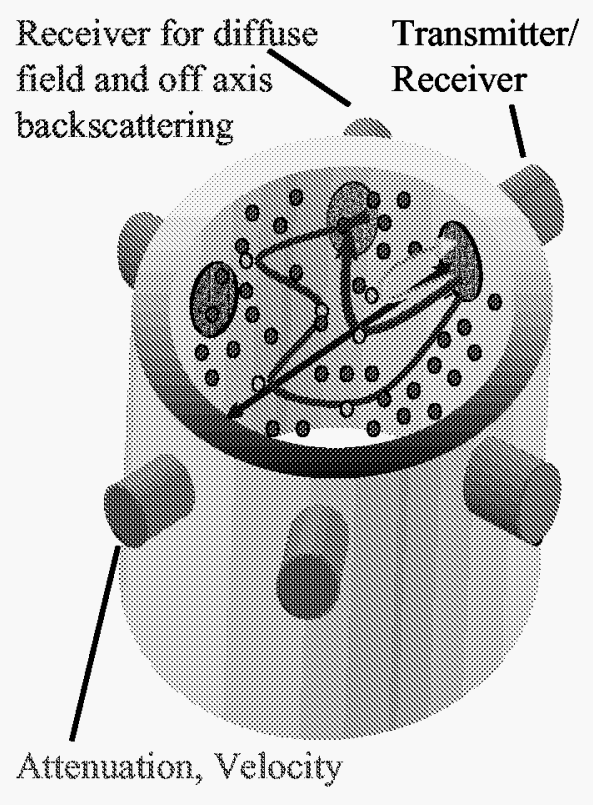

(b) Attenuation and Velocity

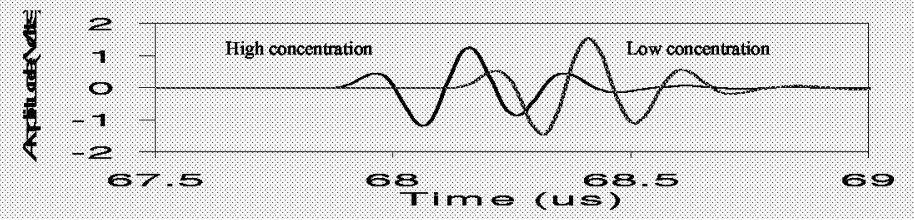

(c) Backscattering (70 um particles)

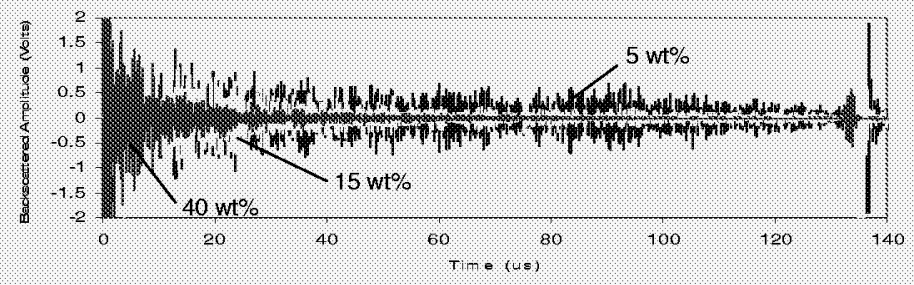

(d) Diffuse field

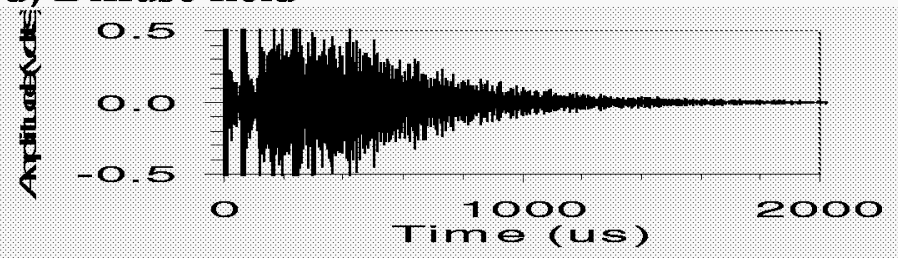

Figure 5. (a) Schematic of ultrasonic measurement apparatus, (b) through transmitted RF signal for attenuation and velocity determination, (c) directly backscattered signal, and (d) diffuse field signal.

To characterize polydispersed specimens, the ultrasonic attenuation as a function of frequency was measured for the $\mathrm{Al}_{2} \mathrm{O}_{3}$ specimens shown in Table 1 . The attenuation as a function of frequency is shown in Figure 6. In addition to the attenuation increasing as the particles were added, the dependence on frequency also changed. The change in frequency dependence can be explained by considering the frequency dependence of the attenuating mechanisms on the product of frequency and particle size as shown in the right side of Figure 6. Specifically, as the average particle size increased, the frequency response increased following the curvature between the frequency to the $1 / 2$ power and frequency to the $4^{\text {th }}$ power. The precise measurements show sensitivity to small changes in concentration and average particle size.

To further characterize polydispersed specimens, a second set of specimens were prepared as described in Table II, where the concentration was held constant wile the particle size distribution was altered. Precise ultrasonic attenuation measurements were performed as a function of frequency to determine the sensitivity to small changes in the particle size distribution. It is significant that the PNNL measurements of the ultrasonic attenuation were made with such high precisions that we were able to measure changes as small as a 1.5 PPM addition of 1 um particles to a slurry of 0.05 um particles. Further measurements utilizing the backscattering and the diffuse field are being performed and will be reported in subsequent reports. 
Table I. Polydispersed specimens where both concentration and the particle size distribution were altered.

\begin{tabular}{|c|c|c|c|}
\hline \multicolumn{4}{|c|}{$\begin{array}{l}\text { MIXTURES OF } 1 \text { um DIAMETER PARTICLES IN A } 5 \text { WT\% } 0.05 \text { um DIAMETER } \\
\text { PARTICLE BASE SUSPENSION }\end{array}$} \\
\hline $\begin{array}{c}\text { Began with } 900 \mathrm{~mL} \text { of } 0.05 \\
\text { um suspension }\end{array}$ & $\begin{array}{l}\text { Number of } 1 \text { um } \\
\text { particles/mL of } \\
\text { suspension }\end{array}$ & $\begin{array}{l}\text { PPM of } 1 \text { um } \\
\text { particles }\end{array}$ & \\
\hline $1 \mathrm{wt} \%$ 1um particles & $5.02 E+09$ & 25.2 & \\
\hline $2 w 1 \%$ 1um particles & $1.01 \mathrm{E}+10$ & 50.6 & \\
\hline $3 w t \%$ 1um particles & $1.53 \mathrm{E}+10$ & 76.7 & \\
\hline $4 w t \%$ 1um particles & $2.06 E+10$ & 103.2 & \\
\hline $5 \mathrm{wt} \%$ 1um particles & $2.59 \mathrm{E}+10$ & 129.8 & \\
\hline $10 \mathrm{wt} \% 1 \mathrm{um}$ particles & $5.39 \mathrm{E}+10$ & 270.1 & \\
\hline $25 w t \% 1$ um particles & $1.53 \mathrm{E}+11$ & 766.3 & \\
\hline $33 w t \% 1$ um particles & $2.18 \mathrm{E}+11$ & 1091.5 & \\
\hline
\end{tabular}

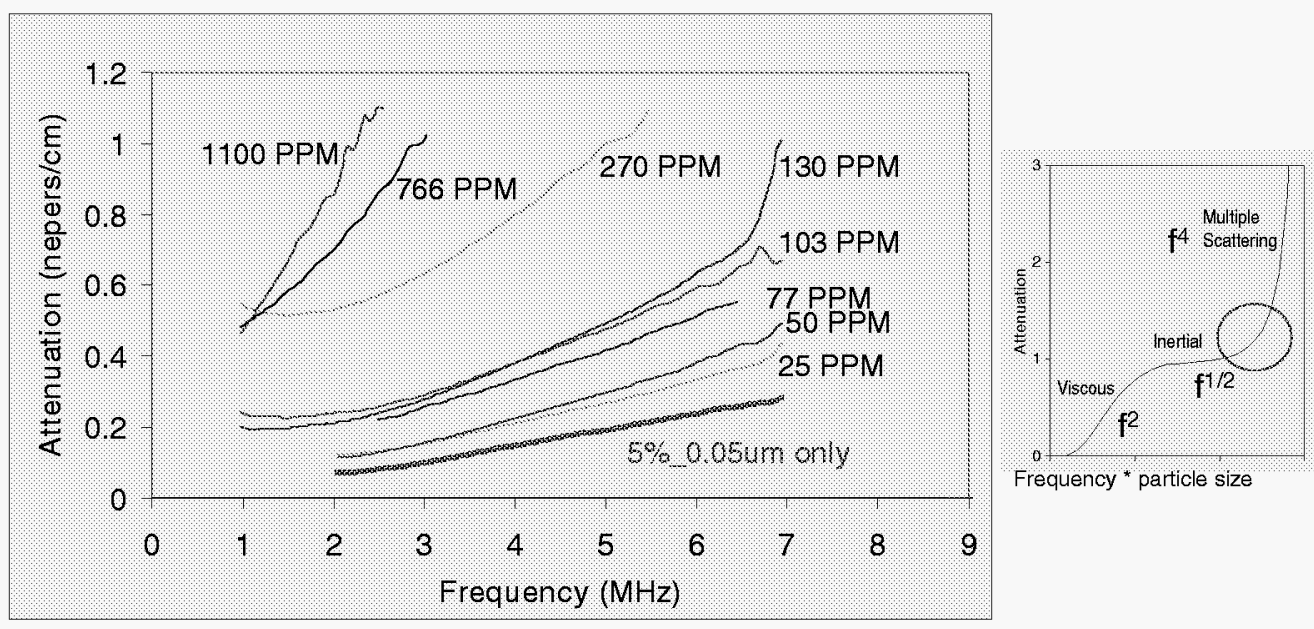

Figure 6. Attenuation as a function of frequency for polydispersed alumina particles in water where both the concentration and the particle size distribution were altered.

Table II. Polydispersed specimens where the particle size distribution was altered while holding the concentration constant. 


\begin{tabular}{|c|c|c|c|c|c|c|}
\hline $\begin{array}{r}\text { vol\% of } 1 \text { um } \\
5 w t \%\end{array}$ & $\begin{array}{c}\text { Number of } 1 \text { um } \\
\text { particles/m } \\
L \text { of } \\
\text { suspension } \\
:\end{array}$ & $\begin{array}{c}\text { Number of } 50 \mathrm{~nm} \\
\text { particles to } \\
\text { every one } 1 \\
\text { um } \\
\text { particle: }\end{array}$ & $\begin{array}{c}\text { Number of } 50 \mathrm{~nm} \\
\text { particles } / \mathrm{m} \\
L \text { of } \\
\text { suspension }\end{array}$ & $\begin{array}{c}\text { Number of } \\
\text { small+large } \\
\text { particles } / \mathrm{m} \\
\text { L now: }\end{array}$ & $\# 1 \mathrm{um} / \# 50 \mathrm{~nm}$ & PPM \\
\hline 1 & $2.98 \mathrm{E}+08$ & $6.61 \mathrm{E}+05$ & $2.00 \mathrm{E}+14$ & $1.97 \mathrm{E}+14$ & $1.51 \mathrm{E}-06$ & 1.5 \\
\hline 2 & $5.27 \mathrm{E}+08$ & $3.70 \mathrm{E}+05$ & $2.00 \mathrm{E}+14$ & $1.95 \mathrm{E}+14$ & 2.70E-06 & 2.7 \\
\hline 3 & $7.52 \mathrm{E}+08$ & 2.57E+05 & $2.00 \mathrm{E}+14$ & $1.93 \mathrm{E}+14$ & $3.90 \mathrm{E}-06$ & 3.9 \\
\hline 4 & $1.03 \mathrm{E}+09$ & $1.85 \mathrm{E}+05$ & $2.00 \mathrm{E}+14$ & $1.91 \mathrm{E}+14$ & 5.39E-06 & 5.4 \\
\hline 5 & $1.28 \mathrm{E}+09$ & $1.48 \mathrm{E}+05$ & $2.00 \mathrm{E}+14$ & $1.89 \mathrm{E}+14$ & 6.77E-06 & 6.8 \\
\hline
\end{tabular}

A part of this project, we are advancing this understanding to better utilize the backscattering and diffuse field measurements to characterize solid liquid suspensions. The backscattering and diffuse field measurements are especially appealing because of the relative simplicity of the measurement and the theoretical description of the scattering processes. Advancements to the measurements performed thus far are being further analyzed to increase our understanding of the mechanisms which govern the ultrasonic scattering and absorption contributions to these measurements. The results are currently being prepared for a patent application. These studies will continue to provide new advancements in the understanding and applications of the ultrasonic backscattering to characterize high concentration solid-liquid suspension.

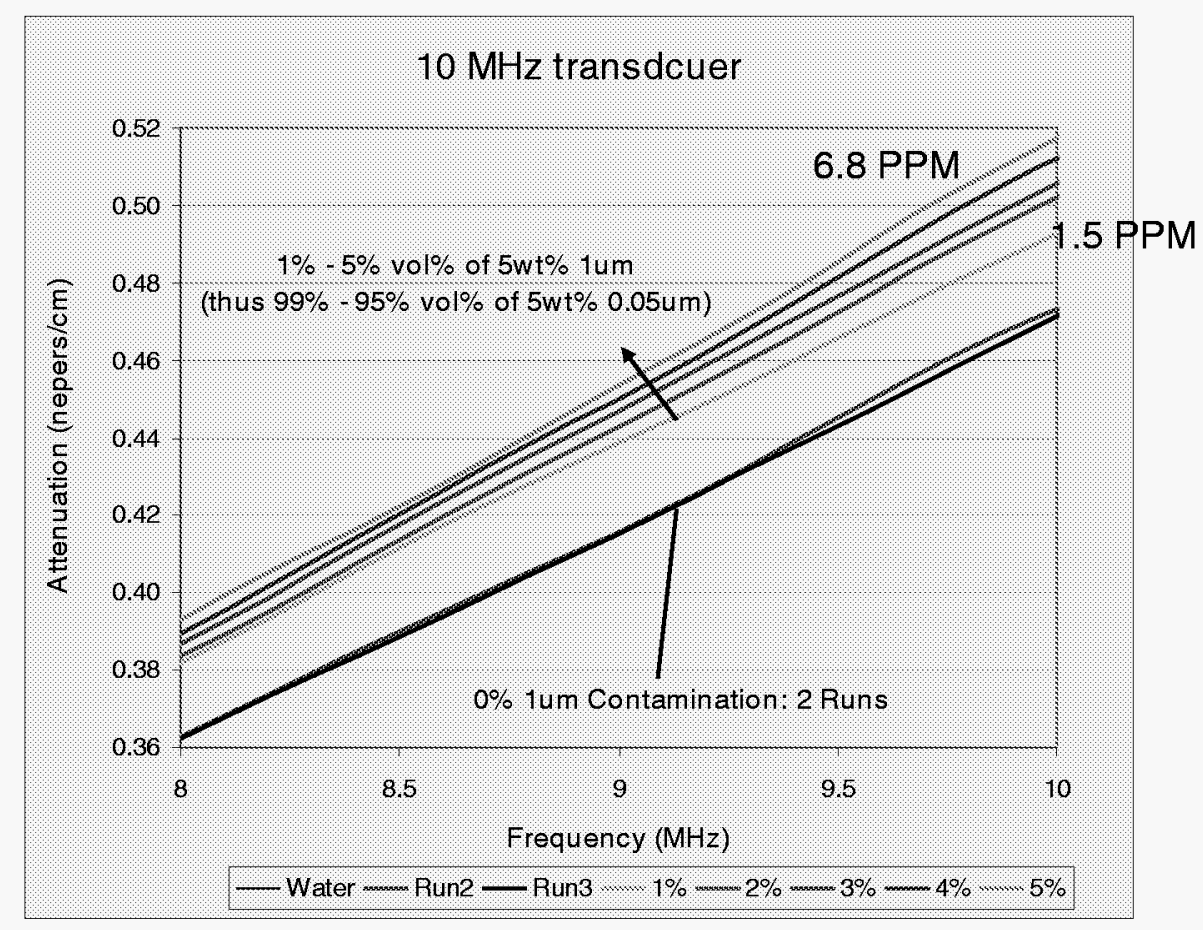

Figure 7. Attenuation as a function of frequency for polydispersed alumina particles in water, where only the particle size distribution was altered. 


\section{Conclusion}

Project research has resulted in numerous presentations at the International Particle Size Analysis 2003 Conference (Harrogate, UK), at the Center for Process Analytical Chemistry Semi-Annual Meetings, at the Review of Progress in Quantitative Nondestructive Evaluation 2002 and 2003, at the International Forum for Process Analytical Chemistry 2004, at the IEEE Instrumentation and Measurement Technology Conference 2003, at the National American Chemical Society Conference in Anaheim 2004, and the Review of Progress on Quantitative NDE, 2004. Publications as a result of this research include Brodsky and Burgess [2003], Randall et al. [2003], Randall et al. [2005] , Panetta et al. [2003], Panetta et al. [2004] , and the Ph.D. Dissertation of Summer Lockerbie Randall [2004].

Disclaimer: Any opinions, findings, and conclusions or recommendations expressed in this material are those of the author(s) and do not necessarily reflect the views of the Department of Energy.

\section{References}

1. Thurber, S.R., A.M. Brodsky, and L.W. Burgess. "Characterization of Random Media by Low Coherence Interferometry." Applied Spectroscopy, 54(10), 15061514 (2000).

2. Brodsky, A., Thurber, S.R., and L.W. Burgess. "Low-Coherence Interferometry in Random Media I. Theory." Journal of the Optical Society of America A, 17(11), 2024-2033 (2000).Thurber, S.R.,

3. Burgess, L.W., A. Brodsky, and P.H. Shelley. "Low-Coherence Interferometry in Random Media II. Experiment." Journal of the Optical Society of America A, 17(11), 2034-2039 (2000).

4. S. L. Randall, A. M. Brodsky, and L. W. Burgess "Manifestation of Mie Resonances in Light Scattering from Multiscattering Media", Inter. J. of Mod. Phys B, V. 19, No. 4 (2005)

5. Randall, S.L., A.M. Brodsky, L.W. Burgess, and R.L. Green. "Optical LowCoherence Reflectometry for Nondestructive Process Measurements," 29th Annual Review of Progress in Quantitative Nondestructive Evaluation (QNDE), Bellingham, WA (2003).

6. Brodsky, A. and L. Burgess. . "Theoretical Study of the Coherent Backscattering of Light by Disordered Media." Intl. J. Modern Physics B, 17(3), 337 (2003).

7. S. L. Randall, "Development and Utilization of Optical Low Coherence Reflectometry for the Study of Multiple Scattering in Randomly Distributed 
Solid-Liquid Suspensions", Ph.D. Dissertation, University of Washington, December, (2004)

8. Golcar, G.R., K.P. Brooks, J.G. Darab, J.M. Davis, and L.K. Jagoda. 2000. Development of Inactive High Level Waste Envelope D Simulants for Scaled Crossflow Filtration Testing, PNWD-3042.

9. Panetta, P.D., B.J. Tucker, R.A. Pappas, and S. Ahmed," Characterization of solid liquid suspension utilizing ultrasonic measurements", 29th Annual Review of Progress in Quantitative Nondestructive Evaluation -2003

10. Panetta, P.D., B. Tucker R. A. Pappas, and S. Ahmed, "Characterization of Solid Liquid Suspensions Utilizing Ultrasonic Measurements" IEEE Instrumentation and Measurement Technology Conference (in press) 